\title{
Electron Beam Induced Deposition and Etching: Fundamentals, Challenges and Nanotechnology-based Applications
}

\author{
J. D. Fowlkes*, D. A. Smith**, M. G. Lassiter** and P. D. Rack**** \\ *Nanofabrication Research Laboratory, Center for Nanophase Materials Science, Oak Ridge \\ National Laboratory, Oak Ridge, TN 37981 \\ ** Materials Science and Engineering Department, The University of Tennessee, Knoxville, TN \\ 37996
}

Correspondence:fo2@ornl.gov

Precise spatial patterning of advanced materials with minimum error is critical for fabrication at reduced length scales. Electron beam induced processing (EBIP) has emerged as a method to define with high spatial precision nanoscale features and elements. However, control of the composition coordinate was proven an elusive challenge when using the EBIP approach for direct-write deposition (electron-beam-induced deposition, EBID). The compositional characteristics of EBID deposits will be discussed, the fundamental reasons for the occurrence of the impurity problem and current approaches to best maximize deposit purity will be discussed. Both EBID as well as the electron-beam-induced etching process (EBIE) require a fundamental understanding of the governing parameter space to obtain the desired, nanoscale end-product. The tremendous and complex EBID/EBIE parameter space includes the local precursor gas flux, primary electron beam energy, electron beam current, surface diffusion rates of adsorbed precursor species, thermal effects on desorption, and the cascade of electron species produced by elastic and inelastic scattering processes. In the case of EBIE, strongly surface bound etch byproducts can significantly rate-limit EBIE efficiency. Characterization of this process for the etching of silicon by electron dissociated $\mathrm{XeF}_{2}$ will be discussed. In this presentation, a variety of experimental studies will be presented to demonstrate the various electron-gas, gas-solid, and electron-solid interactions that are relevant to the electron beam induced processing technique. Reaction rate-limited and mass transport-limited EBID growth modes will be discussed in terms of their respective effects on deposition rate and deposition efficiency in atoms deposited per electron. A well developed computer simulation based on Monte-Carlo calculation sequences will also be presented and compared to various experimental observations. Lastly, several nanoscale device applications will be demonstrated including EBID lithography, scanning probe tip editing, and other nanotechnology-based applications. 

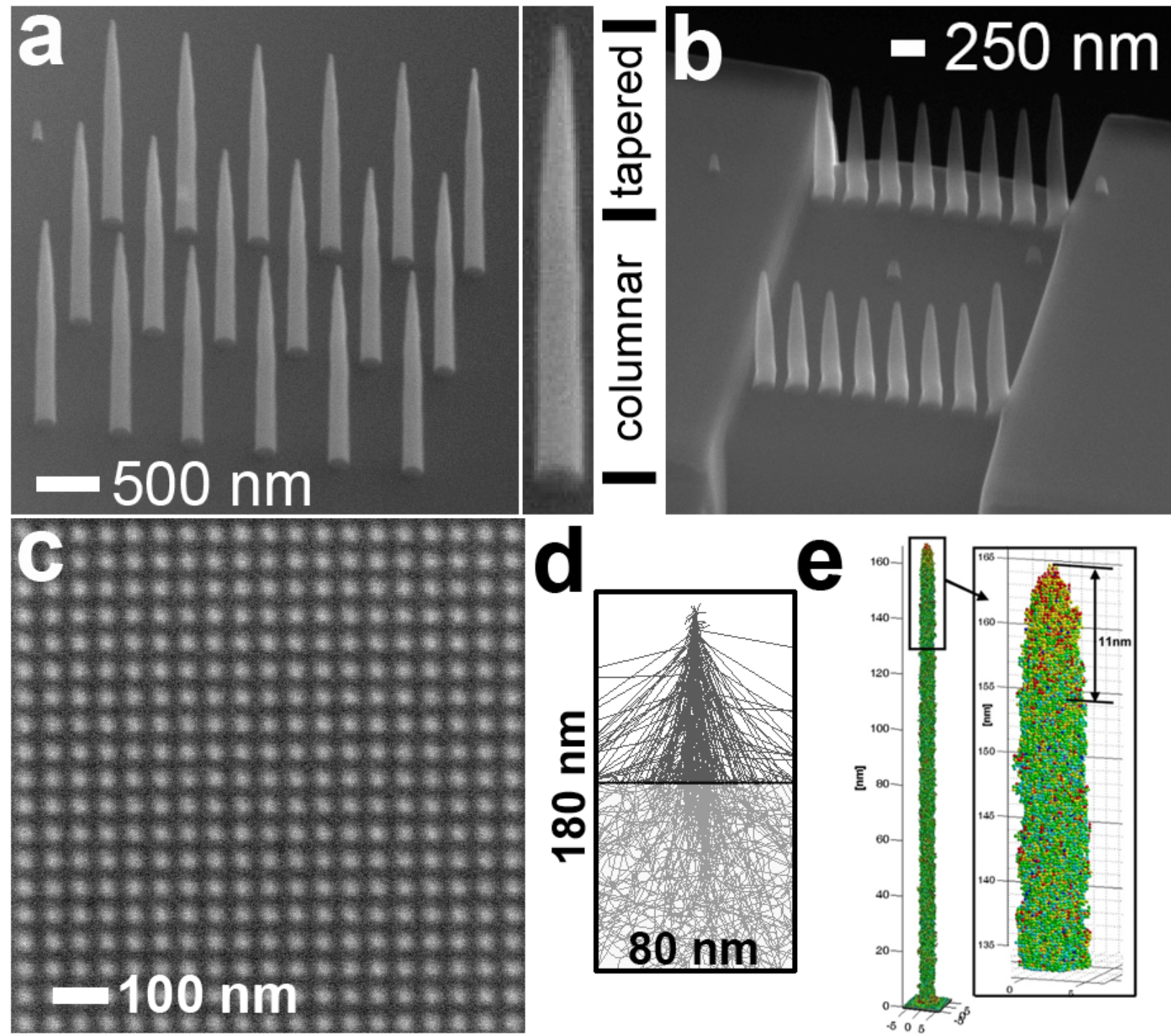

Figure 1 a) An array of nanopillars grown by EBID. A typical EBID nanopillar has a columnar base with a tapered apex shaped primarily by the electron probe accelerating voltage and precursor surface coverage. b) Bottom-up, direct write EBID nanopillar lines serve as the membrane structure in a nanobiotechnology device. c) EBID defined dot array for a subsequent dry lithography step in a nanofabrication process; compositionally pure dots will lead to better etch selectivity. d) A Monte Carlo simulation of 300 electron trajectories scattered in a $100 \mathrm{~nm}$ tall EBID nanopillar ${ }^{15}$. e) Simulation of EBID nanopillar growth where individually deposited atoms have been color coded according to the electron species (secondary electrons, backscattered electrons, primary electrons, and forward scattered electrons) that induced the precursor dissociation and subsequent atom deposit. 